\title{
Experiential learning not enough for organ procurement surgery: Implications for perioperative nursing education
}

\section{ABSTRACT}

Context - Perioperative nurses play a vital role in assisting in organ procurement surgical procedures which occur at the time of a donor's death. Due to limited exposure not all perioperative nurses have prior education or experience in assisting in these surgical procedures. It is well recognised that perioperative nurses receive little targeted education related to the organ donation process and the organ procurement surgical procedure apart from on the job experiential training. Consequently, they are not knowledgeable on all aspects of the organ procurement process and what is required of them at the time when they are asked to participate in these procedures.

Objectives - Within an Australian context and as part of a larger study, this paper describes and presents issues that hindered perioperative nurses' participatory experiences as a result of lacking education, previous exposure and preparation for assisting in organ procurement surgical procedures.

Design - The qualitative grounded theory method was used to develop a substantive theory of perioperative nurses' experiences of participating in multi-organ procurement surgical procedures.

Participants - Thirty five perioperative nurses who had experience in organ procurement surgical procedures from regional, rural and metropolitan hospitals of two Australian states, New South Wales and Western Australia participated in the research.

Results - Levels of knowledge and experience emerged from the data as an influencing condition reported to impact on the perioperative nurses participatory experiences when assisting in procurement surgical procedures. Six components of levels of knowledge and experience were identified and are described within this paper.

Conclusion - The findings from this study provide a unique contribution to the existing literature and provide an in-depth understanding of the educational needs of perioperative nurses in order to successfully assist in multi-organ procurement procedures. The findings highlight the need to provide more targeted education specific to the needs of perioperative nurses. In particular, education on the process of organ donation, the surgical procurement procedure and care of the organ donor throughout the perioperative phases with an emphasis on the operating theatre setting. These findings have the potential to guide further research with implications for clinical initiatives or education programs specifically targeting the perioperative nursing profession both locally and internationally.

\section{INTRODUCTION \& BACKGROUND}

The need to acquire organs for transplant and a growing public awareness towards the benefits of organ donation has seen a gradual increase in organ procurement procedures conducted internationally and more recently within Australian operating rooms (ANZOD, 2012; Smith, 2012). Whilst research in the last two decades has focussed on boosting organ donation rates and ways to better enhance organ preservation rates there is sparse evidence of how health professionals involved in these surgical procedures are trained and educated on aspects of the donation process and the actual procurement surgical procedure (Lopez-Montesinos et al., 2010; Smith, 2012; Smith et al., 2015). Whilst the demand for organ supply and procurement procedures continues, the importance of educating health professionals is key to the overall success of these procedures.

Numerous international studies have acknowledged the need for more specialised education (theoretical and practical based) for health professionals closely involved in the organ procurement process and surgical procedure (Essman and Lobovitz, 2005; Gupta et al., 2014; Lopez-Montesinos et al., 2010; Rios et al., 2007; Tokalak et al., 2005). Education and training of health professionals closely involved in organ donation and transplantation continues to be a problem not only in Australia but internationally, with reports of insufficient or no education or preparation provided to health professionals (Collins, 2005; Jelinek et al., 2012; Meyer, Bjork \& Eide, 2012; Smith et al., 2015; Tokalak et al., 2005). Several authors have presented research evidence both quantitative and qualitative 
exploring health professionals education and knowledge related to the organ donation and procurement process with results indicating that healthcare professionals are often uninformed or lacked knowledge on aspects of the organ donation process, the procurement surgical procedure or brain death diagnosis (Collins, 2005; Essman and Lebovitz, 2005; Jelinek et al., 2012; Kim, Elliot \& Hyde, 2004; Marck et al., 2012; Meyer et al., 2012; Smith, 2012; Rios et al., 2007; Tokalak et al., 2005; White, 2003). Collectively, these research studies have identified the need for increased knowledge, clinical education and training in order to ensure health professionals are knowledgeable on all aspects of the organ donation processes thereby enhancing positive attitudes towards these procedures (Collins, 2005; Essman and Lebovitz, 2005; Kim et al., 2006; Rios et al., 2007). Several authors have documented the need for all professionals in the process of organ donation and transplantation to continue engaging in continuing education and to update their knowledge and clinical skill base as these procedures are seen as demanding and emotionally challenging (Essman and Lobovitz, 2005; Lopez-Montesinos et al. 2010; Rios et al., 2007; Tokalak et al., 2005).

From a perioperative nursing context, it is acknowledged worldwide that perioperative nurses assist in organ procurement and transplantation surgical procedures, usually acquiring skills to participate through informal clinical mentorship and experiential learning at the time these specialised procedures are required (Carter- Gentry and McCurren, 2004; Regehr et al, 2004; Smith, 2012; Wang and Lin, 2009). Nonetheless, the literature confirms that there is a dearth of research or anecdotal evidence of how the perioperative nursing profession is trained to undertake these surgical procedures, their levels of knowledge of these procedures and the process of organ donation (Smith, 2012; Smith et al., 2015). For perioperative nurses' attributing knowledge and education towards procurement surgical procedures is largely undertaken within the clinical environment of the operating theatres (Smith, 2012; Smith et al., 2010). Research studies globally have provided evidence of the traumatic experiences of perioperative nurses when involved in these procedures and their abilities to cope clinically (CarterGentry and McCurren, 2004; Lloyd-Jones, 1996; Page, 1996; Perrin et al., 2013; Regehr et al, 2004; Smith, 2012; Wang and Lin, 2009; Wolf, 1991; Wolf 1994). Whilst other authors have written articles highlighting what the perioperative nurse should expect when participating during the procedure, the instrumentation requirements in order to conduct the surgical procedure (Fox, 1999; Lilly \& Langley, 1999; Watkinson, 1995) or their own personal account of their participation (Hagan, 1997).

Recent Australian research (Smith, 2012) specific to the perioperative specialty, identified that perioperative nurses largely undertook these procedures via experiential learning throughout their careers with no formal training and knowledge on the organ donation process and surgical procedure which made a significant impact on their overall experiences. This research has also validated that there is no formal or consistent education approach to education for the perioperative nursing profession from one health care facility to another and from one state to another Australia wide other than continued experiential learning (Smith, 2012; Smith et al., 2010; Smith et al., 2015). One can ask is this enough preparation for such an integral and important role? This paper presents the findings from a larger study (Smith 2012) relating to the impact of lacking education and knowledge on perioperative nurses and the overall effects on their performance and abilities to feel comfortable on a professional and personal level.

\section{METHODS}

\section{Study Design}

The grounded theory method (Glaser, 1978; Glaser and Strauss, 1967) was used to explore the experiences of perioperative nurses participating in multi-organ procurement surgery from two Australian states; New South Wales and Western Australia.

\section{Research Sample, Setting \& Recruitment}

The research study was advertised via research flyers where thirty five perioperative nurses (33 females and 2 males) were recruited to the study from two Australian states; New South Wales (NSW) $(n=12)$ and Western Australia (WA) $(n=23)$, from a broad cross section of clinical areas in metropolitan, rural and regional areas. Participants were required to meet the initial inclusion criteria of previous participation in at least one multi-organ procurement surgical procedure and were recruited based on a 
purposive and theoretical sampling method. An information sheet was provided to each participant outlining the purpose of the study with informed consent obtained prior to each interview. Participants who informed the study were predominantly experienced perioperative nurses with professional experience ranging from three to 39 years. Participants' ages ranged from 27 to 64 years with the majority of participants 40 to 50 years of age.

\section{Ethical Approvals}

Ethical approval was obtained from the relevant university Human Research Ethics Committee and the participating health services Human Research Ethics Committees. In NSW, recruitment of participants took place after the study was advertised via a flyer which enabled participants to contact the researcher independent of their health services. Formal written consent was obtained from all participants and confidentiality was ensured by de-identifying each participant with an allocation of a numerical number. The researcher ensured that participants were protected from any undue harm or distress relating to recounting their experiences by disclosure of a counselling support should they need this service. During the study no participants sought or required this service.

\section{Data Collection and Analysis}

Data were collected from six sources: participant demographics; semi-structured interviews; the use of memos; reflective journaling; diagrams and conceptual models and the use of existing literature and documents pertinent to the subject area. The interviews were semi-structured and openended in order to obtain rich experiential data with interviews ranging from 30 minutes to 130 minutes in length, with an average of 60 minutes. Interviews were transcribed verbatim and together with the researcher's memoing, were analysed and organised into codes and categories (Glaser, 1978). These codes and categories were further compared and contrasted for similarities and differences with other participant's interviews. Theoretical sampling refined the open ended questions as these became more focused leading to a saturation of the data when no new information was being supplied by the participants.

\section{RESULTS}

Using the grounded theory method, the substantive theory "finding meaning to overcome hiding behind a mask" was identified from the data (Smith, 2012). The process of identifying the main categories and a schematic diagram of the theory has been published previously in whole (Smith, 2012; Smith et al., 2015). Within this substantive theory, levels of knowledge and experience was identified as an influencing condition of the study participant's experiences of both hiding behind a mask (the basic social psychological problem) and their ability to be finding meaning (the basic social psychological process) as a result of their participation in multi-organ procurement surgical procedures (see Figure 1). Four components of levels of knowledge and experience were identified as influencing the problem of hiding behind a mask: 1) participants' prior experience and exposure to procurement; 2) lacking professional development opportunities; 3) lacking education resources and 4) limited mentoring opportunities. Two components of levels of knowledge were identified as influencing the process of participants finding meaning: 1) Having prior knowledge and experience with procurement procedures and 2) Sharing knowledge through team mentoring. 


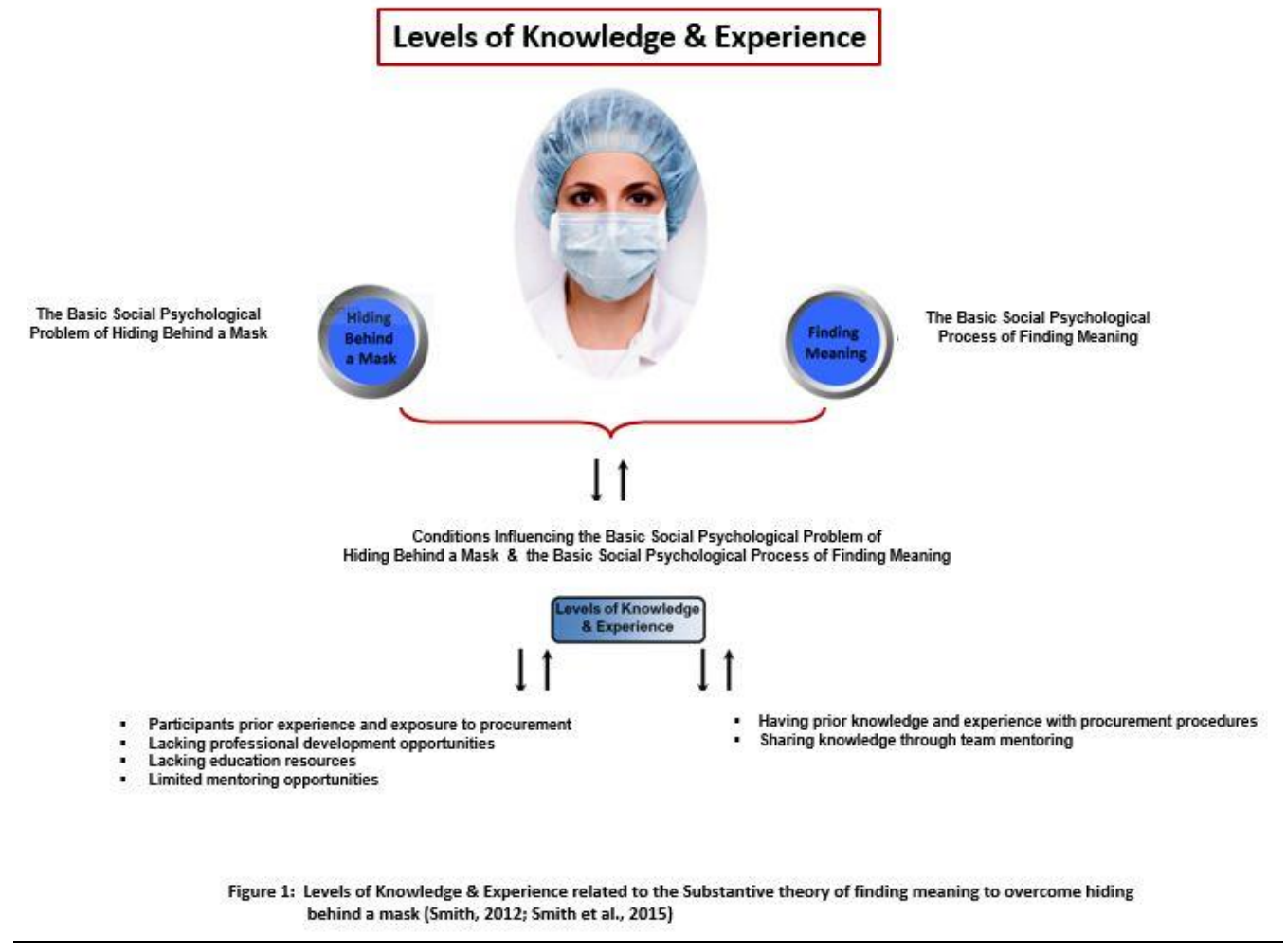

To complement the qualitative data, demographic data revealed the limited sources of education provided to the participants in order to undertake their roles in these surgical procedures. It was noted that 26 of the 35 participants had no formal education provided to them prior to their participation, 6 participants had some form of education via on the job training and two participants obtained education via a hospital in-service whilst one participant was provided information about organ donation at a perioperative course overview.

\section{Participants' prior experience and exposure to procurement surgery}

Participants' prior experience and exposure to procurement surgery was reported as a major issue in their ability to manage these emergency procedures at the time they presented to the operating room. Several participants reported not having had any preparation through prior exposure to procurement surgery at the time of their first participation: "I was overwhelmed, like "my god what am I doing?"(P16). Many nurses viewed these procedures as difficult to master for both experienced nurses and inexperienced or junior perioperative nursing staff. One nurse emphasised this point stating: "I think it would be very difficult for nurses with limited theatre experience to cope with, they would probably need a greater level of support and preparation to be involved in those cases" (P13). Hence the majority of participants expressed the view that some years of post-registration experience within the perioperative nursing setting was essential prior to undertaking such procedures in order to meet the surgical demands and expectations of local or visiting procurement surgical teams.

Several participants reported that not all experienced perioperative nurses had exposure to organ procurement procedures especially if they had previously worked in smaller or private hospitals who were not undertaking such procedures. Although these nurses may have had several years of perioperative nursing experience these nurses voiced similar concerns: "After a long career in theatre it had a profound effect on me ... it was a tough experience for me" (P26). Other participants explained that regular exposure to these procedures was required to reinforce their knowledge and experience of the procedure as it took time to develop their clinical knowledge and skills with a major barrier being the length of time between exposure to these procedures in order to obtain current and up to date professional experiences. One nurse explained: "We don't do enough [multi-organ procurement surgical procedures]" (P16). Some hospital facilities undertook more of these procedures than others 
which provided limited opportunities to perioperative staff: "We don't do many here at *[Name of Hospital] so when we do have them everybody is not really quite sure what to do" (P31).

An important insight expressed by several of the participants was the fact that regardless of their prior levels of perioperative nursing experience and number of times exposed to these surgical procedures they continued to report difficulties mastering these procedures as each donor's procedure was described as uniquely challenging with different experiences and issues always presenting within the clinical environment. One nurse described this as: "I think that every case is different in that you are taking different organs [from each donor]" (P12). Similarly this same viewpoint was highlighted by another participant who commented:

Everything [every procedure] is very different. We did [a multi-organ procurement surgical procedure on] this older man, he had a stroke of some sort ... it was just a different situation compared to the young boy [donor], compared to the mother [donor] who was going to have a cardiac death [DCD] retrieval so [I experienced] three very different situations [and surgical procedures]. (P27)

\section{Lacking professional development opportunities}

Participants within this study articulated that there were lacking professional development opportunities provided by their healthcare facilities including no formal education programs to assist perioperative nurses to gain specialist knowledge and skills in assisting with these surgical procedures. This also entailed no formal opportunities for professional career development and continued learning within this expanding and specialised area. One participant emphasised this point by her comment:

Well, I hadn't had any preparation for it [assisting in procurement procedure] at all and it's not really talked about or anything and no sort of in-services were given in the actual theatre [in] my experience and I'm not sure if there has been anything formally done since. (P24)

Within the clinical environments of various healthcare facilities, this study uncovered the widely held assumption that perioperative nurses did not require specialised education, training or professional development in this area as it was perceived that if a perioperative nurse was capable of undertaking a basic laparotomy then they could successfully undertake organ procurement procedures. This view was validated by one of the participants as: "It was more I have the skills to do a laparotomy therefore I have the skills to do [an] organ retrieval because it was just another level of the [surgical] process. It's no different from doing an anterior resection" (P8). Participants in the study who held staff development or educator positions also substantiated this by: "No we don't do formal teaching [for procurement]" (P2).

Participants emphasised the importance of their role to the overall outcomes of the procurement surgical procedure and believed that formal education was the key:

I think the [perioperative] nursing role needs to be looked at in so far as recognising that it's important in the way of having teams that can do it [the procurement procedure] and more education [for perioperative nursing staff] instead of it is [being viewed as] just another scrub or another scouting role because it's much bigger than that. (P18)

Whilst, another participant summed up the importance and necessity for more education in order to reduce and alleviate negative initial experiences towards participating in these procedure:

I think education is so important. If I'd had some [procurement] education prior to doing my first procurement surgery I would have probably found I felt a lot better about it afterwards because at least ... it was like okay so this is what it involves, I had no idea before that, no idea until I got a phone call in the middle of the night and had to come in and do it [assist in a procurement procedure] ... we need more education. (P19) 
Another area where they felt they lacked education and preparation was how to care and communicate with the donor patient's grieving family within theatre. Many nurses conceded that they lacked experience in sharing information from a surgical context about the nature of the procedure: "There are a lot of things that happen when dealing with [bereaved] families ... outside the operating theatre that we have no understanding of" (P12). In addition, nurses acknowledged they lacked confidence in managing the donor's bereaved family within theatre what to say and what not to say: "I have absolutely no idea about how to deal with relatives, how they are approached about organ donation how much [information] they are told about what goes on" (P4).

\section{Lacking education resources}

Lacking education resources was identified as another component of levels of knowledge and experience. Organ procurement resources, educational materials or courses were reported as limited and difficult to obtain within all healthcare settings across the metropolitan, regional and rural areas. Although, it was perceived that nurses working in the larger metropolitan areas would have better access to education and greater opportunities for experiential exposure to procurement procedures along with training opportunities, according to participants this was not so. One participant made the following comment:

Considering I've worked and spent a lot of time at two of this state's major trauma hospitals I've not once had any education on organ procurement apart from your little talk [providing a background to the study] when you came, that is the first time it has come up. I had to seek out all of my own information ... I have sought out [information] myself no, there has been no discussions beforehand [prior to the procedure], there is no discussions after and there is no in house education which is a bit of a shame. (P6)

The participants from the regional and rural areas had to also contend with other factors such as a lack of clinical exposure to a range of complex or specialised surgical procedures often not undertaken within their theatres. An example was the specialty of cardiothoracic surgery, where several nurses had not had previous experience and exposure to the techniques of opening the chest cavity in order to expose the heart and lungs: "When I've assisted in the retrieval, more like the cardiac [and] thoracic part[s] of the procedure that was new to me" (P12).

Education resources towards organ procurement for the perioperative nursing speciality were reported as predominantly hospital in-services by organ donor coordinators on the peripheral aspects of organ donation which did not cover the procurement surgical procedure itself. Only a few participants reported attending such an in-service program: "No, just attended [an] in-service" (P32). Whilst another younger participant spoke of attending an in-service session organised through a post graduate course specialising in the perioperative nursing field: "No, just an in-service during the peri op course" (P21). Several of these participants stated that although these in-service sessions provided some background information on organ donation it did little to assist them in conducting their intraoperative roles: "We only had a lady come recently [she] came and give us an in-service but that was just about saying that it [organ donation] was a good thing" (P25). This view was also reported by another perioperative nurse by the following comment:

We have had one of the donor co-ordinators come to us ... and give us a talk about their role, our role in [procurement] and talking about organ donation and the criteria for ... being able to donate organs and tissue and also ... what's involved in their [the donor coordinators] role when they're not actually ... involved in organ procurement ... as well as ... their promotional type work. (P15)

Several participants discussed the lack of in depth information available concerning for the perioperative specialty as any education provided was not specifically targeted to their specialty needs. One nurse, who was affected by her participation experiences in procurement surgery, spoke of 
attending such and in-service where an organ donor coordinator in her view had glossed over the operative procedure and the psychosocial aspects and effects of participation for perioperative nurses:

I went to, it felt like a re-education class with the donor co-ordinators where they were saying to the nurses, giving them a pep talk because they heard that negativity from the nurses was not good or they needed more positivity from nurses to encourage people to think about donation. It was just the ... same white washing propaganda this woman [the organ donor coordinator] said "You know some nurses actually think that it's a mutilating surgery and it's not at all!" and I just, I gagged and I said "Excuse me, I have spent my entire life in theatre and I've seen everything opened up and pummelled and sutured what have you seen? ... I've never been more traumatised by such a mutilating experience." (P26)

As a result of lacking education resources, many participants reported seeking their own learning opportunities by resorting to reading policy or procedure manuals about organ donation and procurement surgery within their department in order to ensure they were assisting in these procedures correctly: "I also read [the] hospital policy, reading [about] cardiac death and brain death ... [to] make sure we do the right things [when assisting in the procedure]" (P32). Another nurse explained referring to her hospital departmental theatre manual when a procedure was imminent:

I was checking the procedure manual because I had never done any [procurement surgical procedures] before so I just wanted to make sure I was a little prepared ... I had none, [no formal education] I've only read the manual ... well, I read [up] ... about cardiac death (DCD) because I didn't even realise you could do that sort of situation [procedure]. So I read a small section in the little booklet on what to do and what it all involved. (P27)

Nurses identified issues with relating to these manuals as they were often out of date and not relevant to current practice. Similarly, the same issue was expressed for surgeon's preference cards detailing the specific needs of procurement surgeons requirements in order for the perioperative nurses to prepare for the imminent procedure: “.... even the preference cards were outdated" (P19).

In Australia, the major form of education provided to healthcare professionals is through the Australasian Donor Awareness Program (ADAPT) which are predominantly run in the metropolitan areas, limiting attendance by participants working in the regional and rural areas due to distance or being released from work duties. Some participants who did attend an ADAPT workshop reported it as useful and beneficial in providing information in order to fill in gaps of knowledge related to organ donation and brain death diagnosis. One nurse explained:

I went to the ... ADAPT workshop and they talked a lot about the brain death and the tests they do and the two doctors that do the tests and all that sort of stuff. So I wasn't aware of all that before. (P22)

More importantly, it was emphasised that although such a workshop was available, the ADAPT course was not specific enough to meet the needs, for example of perioperative nurses and their respective intraoperative roles:

I did attend a study day though, run by the ... national donor program (ADAPT). It's one they go to each state and they do it just once a year. I did go to one of those sessions but it was mostly for ICU staff, which covered the family and brain stem death testing and all that sort of thing but [there was] nothing [no specific information] for perioperative [nurses] or any sort of training basically. (P9) 


\section{Limited mentoring opportunities}

Limited mentoring opportunities was reported by the participants as another component of levels of knowledge and experience, where there was limited opportunities for nurses to obtain experiential learning with a mentor within these procedures. Several nurses explained that the lack of mentoring opportunities compounded their ability to obtain practical on the job guidance in procurement surgery in a safe learning environment. It was further identified that, not having regular mentoring opportunities exacerbated the participants' need to hide their lack of knowledge and experience when they were required to assist in a procedure. Within the operating room mentoring is common practice and is undertaken during clinical exposure to different procedures. A common metaphor verbalised within the perioperative setting when participating in a surgical procedure for the first time is: "see one, do one, teach one" (P5; P24; P29). However, for procurement procedures, participants were often put in a position of having to assist with the surgical procedure often before they had had an opportunity to either see one or be mentored clinically. Several nurses expressed that following their initial procedure it was a common expectation that they were then ready to provide mentoring in this area if the situation arose to other staff members.

Factors that affected mentoring opportunities were reported as decreased staffing levels, a lack of experienced staff on the day to assist in the procedure, let alone take on the mentoring role: "Yes, I was anxious and scared because nobody [the nursing team] knew what we were doing" (P31). Increased workloads, the time to conduct mentoring and being asked to participate in procedures that occurred sporadically and quite unexpectedly also hampered efforts to mentor staff. One participant highlighted this point:

[There] could be a bit more in the training side of things or preparation in ... multi organ [procurement procedures], but then I don't know how they would fit that [education] in you know. You do rotations in this place *[Name of Hospital] and you never get to actually spend time in your [own surgical] specialty anyway due to extra workloads ... you're constantly [working] in other [theatre specialty] areas. (P5)

Lacking staff numbers, in addition to issues of staff retention and turnover of staff within particular theatre environments meant fewer experienced staff were available to provide mentoring towards these procedures. This was supported by the following nurse who explained:

No, because most of the time you find that because we have such a high turnover of staff there is always very seldom someone there that has done it before [participated in a procurement procedure] or they can really explain it [the procedure] ... you know it's not something [a procedure] that happens all that often, you know. (P14)

The time critical nature and urgency of these procedures often limited the opportunity for mentoring to take place, with several nurses reporting a quick verbal overview of what was about to take place: "I had nothing [no education or mentoring prior] to begin with just a senior nurse who said "We're harvesting, this is what happens and this is how it goes and this is what you need to do." (P30). Participants reported that due to these limited mentoring episodes they were often left on their own to manage the procedure, having to hide their lack of knowledge and get by with what they knew: "You go out on your own [when participating], you know you have got nobody else to fall back on when you are working with inexperienced staff" (P23).

Surgeons were also reported as integral in the mentoring process of perioperative nurses during the intraoperative phase however they were not always available to offer full support and mentoring during the busy procurement procedure. Mentoring which took place by surgeons was reported in the form of intraoperative instructions and prompts on surgical instruments throughout certain stages of the procurement procedure. One nurse emphasised the importance of requiring such support and assistance with the instrumentation requirements at the time of opening the chest and excision of the heart and 
lungs:

I required mentoring [by the surgeons] especially when they took the heart and lungs as I'd never done cardiothoracic surgery. They took the saw and [the things] they would need so I had to have it ready on the table. If I'm busy they could just grab it. (P9)

Importantly, it was noted that surgeons would provide limited mentoring of perioperative nurses as they were also often mentoring their own junior medical colleagues during these surgical procedures: "Most of them [the surgeons] are very [busy], they talk to each other more so than [they] talk to us [the perioperative nurses] and they'll explain the anatomy or something like that to each other" (P25). Similarly another participant disclosed: "The cardiac surgeon was ... explaining the procedure to the medical student about what he was doing ... the surgeon was also teaching the junior reg [surgical registrar] as well" (P21).

The organ donor or transplant coordinator were also reported to provide some forms of mentoring to perioperative nursing staff as these health professionals were often in the theatre at the time procurement procedures were being undertaken. However these mentoring opportunities were often limited, as the coordinators were usually undertaking their own professional roles such as assisting with coordinating the various procurement teams, completing necessary paperwork and focussed on the appropriate packing of the organs procured for urgent transportation. Whilst this was the case they still ensured they provided some levels of guidance or mentoring to the perioperative nursing staff. Participants described this mentoring as limited however immensely helpful throughout certain stages of the procedure as she/he had their own duties to fulfil. This was emphasised by one participant as: "So we were all fairly new and most of the guidance came from the donor co-coordinator ... we had no one else but they were not always available" (P14).

\section{Having prior knowledge and experience with procurement procedures}

Nurses who had worked in the operating room environment for several years who had some prior exposure to procurement procedures reported increased abilities to cope with the technical nature and the emotional demands of assisting within these surgical procedures. This was expressed by one nurse as: "[Perioperative] nurses with a degree of previous or prior [procurement] surgery experience would be able to cope better ... with these cases in whatever roles scouting or scrubbing" (P13). Those participants working in the metropolitan areas also reported higher levels of knowledge and experience due to the increased frequency of these procedures being completed in metropolitan hospitals: "I have been involved with multiple organ procurement [surgery] ... multiple times probably approximately ten times all here at *[Name of Hospital]" (P12). Whilst participants from the rural or regional areas continued to acknowledge having less experience due to the limited nature of exposure to these procedures. This situation thereby lessening their ability to be finding meaning following their participation as they still struggled to come to terms with their participation: "I've been involved in four multi-organ procurement procedures and it took time to come to terms with my participation in each of those procedures" (P28). Further participants explained that each experience made their subsequent participation process a little easier as they were able to establish ways to tackle the technical and sequential stages of the procedure: "The second one I knew ... the sequence of events" (P14); "It was better the second time I participated. I had a better understanding of what was happening [the procurement surgical process] because I had such a horrible experience the first time" (P18).

To complement their clinical skills several participants explained that their drive to increase their level of theoretical knowledge on the organ donation process was a result of self-directed learning in the area by undertaking a professional development workshop such as the Australasian Donor Awareness Program (ADAPT). One nurse commented:

Yes, I went to the ADAPT program, I did that because I wanted to know more and I think that is the only thing I did ... I think it was a single day course, a full study day but 
that was well after I had done probably the majority of retrievals or organ procurements that I've actually done already and I chose to do that. (P16)

The same nurse further emphasised the benefits from attending such a workshop as increasing her overall awareness and knowledge about the organ donation process in general:

I'm much more comfortable with it [assisting in the procurement surgical procedure] now because before ... I didn't have an awareness of what was exactly going on throughout the whole procedure and now with my education and training I have a greater understanding. (P16)

\section{Sharing knowledge through team mentoring}

Although participants found these procedures difficult they articulated that they found sharing their knowledge through team mentoring helpful to their overall participation and finding meaning from their experiences. This gave participants a purpose and an opportunity to work through their own issues whilst also sharing their own knowledge and experiences with their peers. Several participants who engaged in mentoring other nurses found this role fulfilling and meaningful. They disclosed they had an opportunity to make a remarkable and positive difference to other nurses' experiences when participating in procurement surgical procedures:

Professionally, I feel that if I can help other people who have never experienced this [procurement surgery] and ... help them be involved and find it a positive experience for them, [then] that's something that I have enjoyed helping others get through the procedure. (P15)

Whilst other nurses also spoke of sharing not only their clinical experiences through mentoring but their knowledge that they had gained through further education and training. One nurse disclosed:

With my education and training, I feel now that I can actually teach the staff that are working alongside me whereas when I first started I was learning a lot myself so I couldn't actually be involved in the teaching role. (P16)

Again within this study, participants highlighted that more should be done to ensure that perioperative nurses had adequate education, training, exposure to these procedures and mentoring opportunities in procurement surgery. One nurse emphasised this point by her comment:

There needs to be education. I think you could do education for these [procurement] cases at different levels and I think some basic education of what to expect would be helpful [to perioperative nurses], whether that [education] would be done internally within the department or whether that could be done by the [organ donor] co-ordinators [when they visit the theatres]. (P13)

\section{DISCUSSION}

This research has uncovered the importance and need for specialised education and professional development opportunities for perioperative nursing health professionals in organ procurement surgery. It can be recognised that levels of knowledge and experience impacts on the perioperative nurses ability to participate successfully in these surgical procedures on both a professional and personal level. While health professionals have a duty to be up to date in clinical practice and be practicing within their scope of nursing practice, in order for perioperative nurses to contribute effectively as part of the team assisting in procurement surgical procedures they require more specialised education and support within the clinical environment. The larger study (Smith, 2012) and this paper has uncovered that perioperative nurses within the context of their knowledge and experience with procurement surgical procedures, 
struggled to obtain relevant and up to date information in relation to these important surgical procedures. Moreover this research highlights that there is no consistent approach to education across Australian states or territories, and individual health care facilities in order for perioperative nurses to receive specific education or professional development opportunities in this expanding area of healthcare.

Not surprisingly, this study has uncovered a major assumption that perioperative nurses are adequately prepared to undertake procurement surgical procedures based on their extensive experience and the prerequisite that they have the basic essential skills to undertake a basic laparotomy. The participants in this study have highlighted that this basic assumption has been to the detriment of their professional practice and personal experiences in procurement surgery and a major factor in obtaining and receiving adequate education. To assist in all surgical specialty procedures, perioperative nurses are trained to pre-empt the needs of the surgeon hence having background knowledge of the anatomy and physiology, in addition to the surgical approach and instrumentation requirements to undertake each surgical procedure they assist in successfully. The assumption that a basic laparotomy prepares a perioperative nurse to undertake a multi- organ procurement surgical procedure has not rung true within the study nor the culture of "see one, do one, teach one". Hence the study participants have emphasised the requirement for more in-depth education.

Several participants reported that they had not had prior experience and exposure to procurement surgery and this was identified as a major influencing condition of participants hiding behind a mask. Participants reported hiding their lack of knowledge and understanding of these surgical procedures when assisting with procurement teams, also feeling uncomfortable taking on the responsibility for teaching and mentoring their fellow colleagues. As noted, many participants themselves felt unprepared for assisting clinically in procurement surgical procedures, lacking knowledge of the procurement process, what was expected of them when participating in these surgical procedures in addition to feeling unprepared emotionally for what they would see and do as part of their roles. Lack of knowledge and experience also extended to the nurses own fears and concerns that they lacked knowledge and understanding of the donor's brain death diagnosis and brain death testing. Several nurses felt uncomfortable when they lacked understanding of the donor's brain death diagnosis (Smith et al., 2015). This was not an uncommon finding as several other authors also indicated health professionals knowledge deficits and doubts related to brain stem testing results (DuBois and Anderson, 2006; Floden and Forsberg, 2009; Sadala et al., 2006; White, 2003).

The incidence of procurement surgical procedures across metropolitan, regional and rural areas also impacts on perioperative nurses and health professionals obtaining experience and exposure to these surgical procedures. As reported within this study, having prior experience and exposure was limited for the majority of perioperative nurses who worked in the rural or regional areas, where they were further disadvantaged by staffing issues, propelling them into these procedures with limited knowledge compared to nurses who worked in the metropolitan areas. The need for health professionals to have some prior experience and exposure was also emphasised in the study by Essman and Lovitz (2005) on medical students who reported the benefits of allowing these students the opportunity to observe the entire donation process from the ICU to the operating room. Several of the participants within this study reported that they would highly recommend this form of education to fellow students as it was viewed as beneficial within the clinical environment.

A major finding, again overlooked within the literature however identified within this study was the nurse's uncertainty in dealing with deceased organ donors within the theatre environment and the psychological effects on them given they usually deal with saving lives and restoring patient's wellbeing through life saving surgical procedures. As noted from the study findings several participants wanted to avoid the fact that they were operating on a deceased organ donor as dealing with death was not a common occurrence within the operating rooms. Unfortunately staff revealed that they did not have the adequate skills or training to deal with such events and felt overwhelmed when dealing with both the donor patient and their respective families. Several perioperative nurses therefore reported that they did not have the knowledge base or skills to talk with these bereaved families, and they tried to 
avoid such encounters. This was evident when nurses reflected that they felt uncomfortable dealing with the families of both brain dead and DCD donors. This study would suggest that within the operating room setting more education is required to prepare these health professionals in order to deal with the bereaved family members of organ donors who come into the operating room environment to see their loved ones either prior or post their surgical procurement procedure.

The need for professional development opportunities and education was emphasised as pivotal to the overall outcome of these surgical procedures on a professional and personal level for the nurses within this study. Several nurses believed that fundamental education, knowledge and understanding provided an opportunity to enhance their skills, knowledge and experience within these procedures positively. As these fundamental resources were lacking, participants described instances where they sought their own learning opportunities by reading policy or procedure manuals about organ donation and the procurement procedure in order to educate themselves. Clearly, further professional development opportunities specific to the perioperative nursing specialty and the intraoperative process of procurement surgery are required as across Australian states and territories from metropolitan to regional and rural areas as educational opportunities are limited or insufficient. As reported, only a handful of participants had received some form of education or training prior to their participation in these surgical procedures and the effectiveness was inadequate for their professional needs. Surprisingly, the majority of participants in this study disclosed that they had not been provided with any forms of education prior to assisting in a procurement procedure. Participants also stated that they were never provided with mandatory education on procurement surgery or organ donation as part of their theatre induction or as part of a program of continuing education usually provided by a theatre educator or staff development nurse within their own health care facility operating theatres.

This study also identifies the dearth of information internationally on specific and targeted education or courses for the perioperative nursing speciality although perioperative nurses make such a large contribution to the success of these surgical procedures worldwide. Education as a whole for the perioperative nurse is described as undertaken via experiential learning at the time these procedures are conducted. How much education and whether this was effective training has been validated by the participants views that experiential learning did not prepare these nurses adequately in order to perform their professional roles within these procedures. If anything this study has emphasised that experiential learning alone is flawed as each new donor and procurement procedure presents different situations, management of donors and experiences for the healthcare team. More recent studies and researchers also support the notion for more in-depth clinical and theoretical education on aspects of organ donation and transplantation (Collins, 2005; Floden and Forsberg, 2009; Kim et al., 2006; Meyer et al., 2012). The current research findings validate that more is needed to be done in order to educate health professionals at the frontline such as perioperative nurses in order to enhance their skills and knowledge when assisting in these surgical procedures.

Clearly, this study has emphasised within an Australian context, that there are currently no effective modes of professional education or specific courses/educational resources for the perioperative specialty related to organ procurement surgery other than the ADAPT course workshops or in-services run by individual healthcare facilities or state organ donation Coordinators. As mentioned although the ADAPT course is designed for all health professionals this did not meet the specific intraoperative surgical procedure and the educational needs of the study participants roles as perioperative nurses when required to assist in such procedures. A major problem identified with this form of education was that not all participants could access this workshop due to staffing issues, release from the work environment and the associated attendance and travel costs to attend a workshop which are usually held in all major Australian cities. Secondly, of those participants who attended such a workshop, the majority proclaimed benefits in attendance by gaining theoretical knowledge and understanding on aspects of the process of organ donation; requests for donation and brain stem death testing however also explained that content was lacking specific to the perioperative environment. Therefore initiatives for perioperative nurses to access education on all facets of organ donation and 
procurement surgery must also take into account the educational needs of perioperative nursing work in addition to the possible high demand of workshop access by all nurses across all Australian regions.

In the current study, increased exposure and clinical mentorship was reported as an excellent form of accessing on the job clinical skills towards procurement surgical procedures. Participants from the current study benefited from this exposure and found these experiences useful however due to limited staffing numbers and opportunities they were not always able to utilise such opportunities. This finding supports the earlier descriptive survey study of perioperative nurses by Lloyd-Jones (1996) only $46.9 \%$ of the study respondents reported some form of teaching during a procurement procedure; however this was also reported likely to not occur due to lack of time and focus on the surgical procedure. This was also a common finding within the current study where mentoring opportunities were often not available due to staffing shortages or lack of time. Initiatives to increase exposure for perioperative nurses through mentoring in procurement surgical procedures would be beneficial as nurses would feel less stress and strain having to manage these procedures on their own and with limited knowledge and exposure. This would in turn help to alleviate the overwhelming clinical skills demands placed on individual nurses at the time of participating in a procurement surgical procedure and would also aid the nurse to address and focus on the emotional demands of their participation which is vitally important to their well-being and their ability to cope. Not surprising, those participants who received mentoring opportunities fared better and reported more positive experience, finding meaning from their participation in these surgical procedures. This also reinforced their ability to share their own knowledge gained to other team members and the opportunity to assist other nurses to more confidently navigate through the procurement procedure thereby promoting more positive participation experiences.

\section{STUDY LIMITATIONS}

The present study contributes to the literature on the need for education towards health professionals such as perioperative nurses by expanding knowledge and providing a perspective from the nurses themselves on what education exists and what more is required by this group of health professionals. A limitation of the study is the relatively small participant sample given that only two Australian states were recruited from therefore it may be difficult to generalise the findings across Australia. The authors recommend that further studies address this by using a larger and more representative sample of perioperative nurses from metropolitan, rural and regional areas across Australia including different types of health care facilities.

\section{CONCLUSION}

Perioperative nurses continue to assist with multi-organ procurement surgical procedures and whilst they contribute in these surgical procedures, more in-depth and intensive clinical education is required in order for these nurses to understand and contribute to these specialised procedures. Clearly, there are challenges with perioperative nurses receiving adequate education towards procurement surgical procedures. This has an impact on their professional responsibilities, personal feelings and attitudes towards their participation. The disparity in the levels of knowledge, experience, exposure and access to these procedures; their ability to obtain experiential learning through on the job mentoring were all identified as contributing to their overall experiences. More importantly, the vulnerability that health professionals feel as a result of lacking education and understanding during these surgical events cannot and should not be ignored (Smith, 2012). The results of this study indicate the need and initiation of comprehensive training and mentoring opportunities amongst perioperative health professionals involved in procurement surgical procedures. Further research is required in regards to; looking at perioperative nurses levels of knowledge related to organ donation and procurement at a national and international level. The incorporation of specialised nurse/roles within the perioperative nursing setting who have the necessary skills and knowledge on procurement surgery to educate and support other nursing staff may be a useful role within health care organisations. The findings of this study have important implications for perioperative nursing education related to procurement surgical procedures and as noted by the current study findings further targeted education for perioperative nurses is vital to ensure that they are adequately prepared and continually trained to undertake these surgical procedures. 
Acknowledgements: The author/s would like to acknowledge the participants who took part in this research and willingly shared their experiences.

Financial Disclosures: Zaneta Smith PhD Funding: (1) 2008 recipient of the Helen Bailey Scholarship (2) 2010 Australian Postgraduate Award (3) 2010 Curtin University Postgraduate Scholarship.

\section{REFERENCES}

Australian and New Zealand Organ Donation Registry (ANZOD Registry). (2012). ANZOD Registry Report 2012. Adelaide, South Australia. Editors: Leonie Excell, Violet Marion, Kylie Hurst, Graeme Russ. Retrieved May 20, 2012 from http://www.anzdata.org.au.

Carter-Gentry, D., \& McCurren, C. (2004). Organ procurement from the perspective of perioperative nurses. AORN Journal, 80 (3), 417-21,424-31.

Collins, T.J. (2005). Organ and tissue donation: A survey of nurse's knowledge and educational needs in an adult ITU. Intensive and Critical Care Nursing, 21(4), 226-233.

DuBois, J.M., \& Anderson, E.E. (2006). Attitudes towards death criteria and organ donation among healthcare personnel and the general public. Progress in Transplantation, 16(1), 65-73.

Essman, C. C., \& Lebovitz, D.L. (2005). Donation education for medical students: Enhancing the link between physicians and procurement professionals. Progress in Transplantation, 15(2), 124128.

Floden, A., \& Forsberg, A. (2009). A phenomenographic study of ICU-nurses' perceptions of and attitudes to organ donation and care of potential donors. Intensive and Critical Care Nursing, 25(6), 306-313.

Fox, R. (1999). Organ procurement: What is our role and what do we need to know? ACORN Journal, 12(3), 19-22.

Glaser, B.G. (1978). Theoretical sensitivity: Advances in the methodology of grounded theory. Mill Valley, California: Sociology Press.

Glaser, B.G., \& Strauss, A.L. (1967). The discovery of grounded theory: Strategies for qualitative research. New York: Aldine de Gruyter.

Gupta, N., Garonzik-Wang, J.M., Passarella, R.J., Salter, M.L., Kucirka, L.M., Orandi, B.J., Law, A.H., Segev, D.L. (2014). Assessment of resident and fellow knowledge of the organ donor referral process. Clinical Transplantation, 28(4), 443-449.

Hagan, A. (1997). Innocence lost in first harvesting. Canadian Operating Room Nursing Journal, 15(1): 31-32.

Jelinek, C.A., Marck, C.H., Weiland, T.J., Neate, S.L., (2012). Organ and tissue donation-related attitudes, education and practices of emergency department clinicians in Australia. Emergency Medicine Australasia. 24 (3), 244-250.

Kent, B.C. (2004). Protection behaviour: A phenomenon affecting organ and tissue donation in the 21st century? International Journal of Nursing Studies, 41(3), 273-284.

Kiberd, M., \& Kiberd, B. (1992). Nursing attitudes towards organ donation, procurement, and transplantation. Heart \& Lung, 21(2), 106-111.

Kim, J.T., Elliot, D., Hyde, C. (2004). Korean health professionals' attitudes and knowledge toward organ donation and transplantation. International Journal of Nursing Studies, 41(3), 299-307.

Kim, J.R., Fisher, M.J., \& Elliot, D. (2006). Attitudes of intensive care nurses towards brain death and organ transplantation: instrument development and testing. Journal of Advanced Nursing, 53(5), 571-582.

Lilly, K.T., \& Langley, V.L. (1999). The perioperative nurse and the organ donation experience. AORN Journal, 69(4), 779-791.

Lloyd-Jones, H. (1996). Attitudes of nurses to donor organ retrieval and visiting surgical teams: The Papworth experience. British Journal of Theatre Nursing, 5(11), 28-31.

Lopez-Montesinos, M.J., Manzanera, J.T., Mikla, M., Rios, A., Lopez-Navas, A., Martinez-Alarcon, L., Rodriguez, M.M., \& Ramirez, P. (2010). Organ donation and transplantation training for future professional nurses as a health and social awareness policy. Transplantation Proceedings, 42(1), 239-242. 
Marck, C.H., Weiland, T.J., Neate, S.L., Hickey, B.B., \& Jelinek. (2012). Australian emergency doctors' and nurses acceptance and knowledge regarding brain death: A national survey. Clinical Transplantation, 26(3), E254-E260.

Melo, J., Batista, A., Teixeira, A., Figueiredo, E., Ribeiro, O., Lopes, P., Pina, J., Carvalho, A.S., \& Granja, C. (2011). Knowledge and behaviour among health professionals in relation to cadaveric organ donation and transplantation: A questionnaire-based analysis in Portuguese hospitals. Transplantation Proceedings, 43(5), 1429-1433.

Meyer, K., Bjork, I.T., \& Eide, H. (2012). Intensive care nurses' perceptions of their professional competence in the organ donor process: A national survey. Journal of Advanced Nursing, 68(1), 104-115.

Page, S. (1996). Response of perioperative nurses to organ procurement surgery. Canadian Operating Room Nursing Journal, 14(4), 9-11.

Perrin, K., Jones, B., Winkelman, C. (2013). The co-existence of life and death for the perioperative nurse. Death Studies, 37(9), 789-802.

Regehr, C., Kjerulf, M., Popova, S.R., \& Baker, A. (2004). Trauma and tribulation: The experience and attitudes of operating room nurses working with organ donors. Journal of Clinical Nursing, 13(4), 430-437.

Rios, A., Ramirez, P., del mar Rodriguez, M., Martinez-Alarcon, L., Lucas, D., Alcaraz, J., Montoya, M.J., \& Parrilla, P. (2007). Benefit of a hospital course about organ donation and transplantation: An evaluation by Spanish hospital transplant personnel. Transplantation Proceedings, 39(5), 1310-1313.

Sadala, M.L.A., Lorencon, M., Cercal, M., \& Schelp, A. (2006). Caring for organ donors: The intensive care unit physicians' view. Heart \& Lung, 35(3), 190-197.

Smith, Z. (2012). Hiding behind a mask: A grounded theory study of perioperative nurses experiences of participating in multi-organ procurement surgery. $\mathrm{PhD}$ Thesis, Curtin University. School of Nursing \& Midwifery, Western Australia. Available from http://trove.nla.gov.au/work/180531201

Smith, Z., Leslie, G., \& Wynaden, D. (2010). Perioperative nurses participating in multi-organ procurement surgery. ACORN Journal, 23(2), 6-13.

Smith, Z., Leslie, G \& Wynaden, D. (2015). Australian perioperative nurses' experiences of assisting in multi-organ procurement surgery: A grounded theory study. International Journal of Nursing Studies, 52(3), 705-715.

Tokalak, I., Emiroglu, R., Karakayali, H., Bilgin, N., \& Haberal, M. (2005). The importance of continuing education for transplant coordination staff. Progress in Transplantation, 15(2), 106-111.

Tuttle-Newhall, J.E., Collins, B.H., Kuo, P.C., \& Schroeder, R. (2003). Organ donation and treatment of the multi-organ donor. Current Problems in Surgery, 40(5), 266-310.

Wang, Y., \& Lin, C. (2009). The experience of perioperative nurses involved in organ procurement. Journal of Nursing Research, 17(4), 278-284.

Watkinson, G.E. (1995). A study of the perception and experiences of critical care nurses in caring for potential and actual organ donors: Implications for nurse education. Journal of Advanced Nursing, 22(2), 929-940.

White, G. (2003). Intensive care nurses’ perceptions of brain death. Australian Critical Care, 16(1), 7-14.

Wolf, Z.R. (1991). Nurses' experiences giving post-mortem care to patients who have donated organs: A phenomenological study. Scholarly Inquiry for Nursing Practice: An International Journal, $5(2), 73-87$.

Wolf, Z.R. (1994). Nurses' responses to organ procurement from nonheartbeating cadaver donors. AORN Journal, 60(6), 968-981. 